\title{
Design of a smart bidirectional actuator for space operation
}

Bortolino Saggin ${ }^{1}$, Diego Scaccabarozzi ${ }^{1 *}$, Marco Tarbini ${ }^{1}$, Marianna Magni1 ${ }^{1}$, Carlo Biffi², Ausonio Tuissi²

1 Politecnico di Milano, Polo Territoriale di Lecco, Via G. Previati 1c, 23900 Lecco (Italy)

2 Istituto di Chimica della Materia Condensata e di Tecnologie per l'Energia, CNR, 23900 Lecco (Italy)

* corresponding author: diego.scaccabarozzi@polimi.it

\section{Abstract}

A common need for space borne instruments, satellites and planetary exploration payloads is the (10) usage of compact, light and low power actuators. In the recent years, this need has been partially solved by the development of customized solutions with an increasing usage of smart materials. A linear bidirectional actuator based on shape memory alloy technology is presented in this work. The device has been conceived to lock the double-pendulum scanning mechanism of a miniaturized Fourier transform spectrometer for planetary observation. The mechanism class is that of pin pullers, with the pin locking the movable components of the spectrometer during launch 6 and landing phases. The proposed mechanism, differently from available off-the-shelf devices, allows multiple actuations without the need of manual resetting. Moreover, the device requires to be powered only to change its status. An appealing feature of the adopted concept is that the actuation is intrinsically shock-less, a key requirement for deployment of devices sensitive to mechanical vibration and shocks. All these characteristics, in addition to the design flexibility of the proposed concept in terms of achievable forces and strokes, make the designed actuator promising for many different applications, from space to ground. The designed bidirectional actuator provides $0.6 \mathrm{~mm}$ stroke and a $50 \mathrm{~N}$ preload but it represents just an example of implementation for the proposed concept. Structural design of the functional elastic components 
and SMA alloy characterization have guided the actuator development. A mockup of the actuator has been manufactured and the predicted performances preliminary validated.

\section{Keywords}

SMA, bidirectional actuator, compliant structure, resettable, pin-puller, holding mechanism, space application, Mars, FTS.

\section{Introduction}

It is well recognized that SMAs provide some advantages in mechanisms and actuators design, i.e. simplicity of related mechanisms, low driving voltage and sensing capability [1]. These characteristics have been recently exploited in different fields, i.e. robotics [2], industry [3] and aeronautics [4] to develop low power linear and rotational actuators. SMAs are very attractive for space applications as well, where SMA technology usage is quite recent $[5,6]$ and mainly focused on the actuation of deployable systems or damping system for spacecraft antennas [7]. In fact, SMAs allow control of the deployment process and thanks to the shock-less actuation have been used for developing low-shock release devices (LSRD). A clear advantage is that no additional damping is required to suppress shocks and vibrations due to the deployment, unlike the systems based on elastic energy storage. Example of space designed LSRD can be found in [8] where SMA and steel springs work in contrast to achieve automatic resetting [9]. Some additional examples of LSRDs can be found in [10-12], where SMA wires are used as triggers for the actuation. Moreover, thanks to the advantages of the SMA technology, some recent customized spaceborne applications can be found in gas release mechanism [13] or rock splitters [14].

5 The advantages of the SMA materials have been also exploited to develop low power linear and rotational actuators [15]. Available spaceborne devices provide mass ranging between 4 and $40 \mathrm{~g}$ and output force between 10 and $50 \mathrm{~N}$. These actuators exploit SMA wire working against bias springs, added to recover the initial configuration once SMA is not heated. This is a relevant limitation for a 
general space application and in particular for instruments mounted on planetary rovers, given that the deployed configuration requires a continuous power consumption.

51 Paraffin based actuator have been used in space as well, thanks to their reliability, large strokes (up to $13 \mathrm{~mm}$ ) and high forces (about $100 \mathrm{~N}$ ). Unfortunately, the actuator mass is not negligible, no less than $80 \mathrm{~g}$. Piezoelectric actuation has been evaluated as well among the existing solutions, thanks to the accuracy of the output motion, the large stuck forces and the compatibility with low temperature and vacuum environment. Anyway, the provided output displacement is generally limited to few microns or, to achieve larger strokes, the actuator mass becomes quite large.

Thus, SMA technology was identified as the most promising for the development of an innovative locking mechanism for the scanning pendulum of a miniaturized FT spectrometer 140x140x120 mm ${ }^{3}$ in size, $1 \mathrm{~kg}$ mass, designed to be mounted on a rover for Mars [16]. Holding was needed to keep the pendulum in a safe position during launch and rover landing phases. This mechanism has become a

61 key component in any proposal for miniaturized FTS that has been conceived since then $[17,18]$. As evidenced by market and literature review, no multiple actuation devices compatible with our design constraints are available, because they are either too massive or exceeding the size and power budgets. Moreover, most of them are qualified down to $-40^{\circ} \mathrm{C}$, an operational limit not compatible with environmental requirements for planetary surface operation. Exomars mission mechanical environment had also challenging requirements due to the expected acceleration peak at landing on Mars. The developed actuator has many positive features: it provides two stable positions, requires power only to change its status, uses only solid lubricants, can withstand at least one thousand working cycles without relevant performances degradation and is capable of working at low temperatures. The

71 actuator concept is provided in the following whereas thermo-mechanical design is presented in 72 Section 2. SMA wire characterization and actuator preliminary testing are provided in Section 3 and 73 Section 4 eventually completes the paper. 


\subsection{Actuator concept}

In order to understand the working principle of the proposed actuator two key points have to be highlighted:

- Actuating force and displacement are provided by SMA wire contraction as consequence of the material heating; and

- The actuator stroke is achieved by means of a displacement amplifier based on selectively compliant element that amplifies the SMA wire contraction without frictional elements.

A sketch of the actuation concept is shown in Figure 1. SMA wire is wrapped around an elastic support of diameter D and since the wire has been previously deformed, thanks to the material memory effect [19], it recovers its original shape once heated. The wire contraction reduces the support diameter and a new configuration (with diameter D') is achieved, resulting in the output stroke provided by the actuator (as consequence, the elastic support extends from L to L'). The concept is simple and reversible, since a pushing force applied to the elastic support stretches the wire to its original configuration once the yield stress is exceeded.

The elastic support design has a key role in the actuator performance since the output displacement and force can be amplified by proper geometry selection. In SMA actuators design, displacement amplification is a recurrent problem, developed mechanisms can be found in various references [20, 21]. Authors in [21] design a passive elastic system to increase the output stroke by about a factor 2.5 and achieve constant force during actuation. Mechanism proposed in literature cannot be implemented in our case, because of our strict requirements of lightness, compactness and avoidance of lubricants. Thus, the wire contraction amplification has been achieved with selectively compliant element described in the next section. A preview of the final geometry is shown in Figure 2 . The elastic supports are derived from a thin disk slotted in eight sectors. Two disks are kept together by an additional elastic structure that allows the initial positioning and avoid unwanted motions of the disk sectors during the actuation. The proposed system has an intrinsic higher stability than planar 
devices and allows the implementation of friction less joints, a mandatory requirement for space and vacuum applications. Thermally and electrically insulating sectors are glued over the cylindrical structure between the wire and the disks. These are cylindrical as well and guide the wires avoiding any axial sliding during the actuation. Finally, two crimping elements are used to transmit the wire load during the actuation.
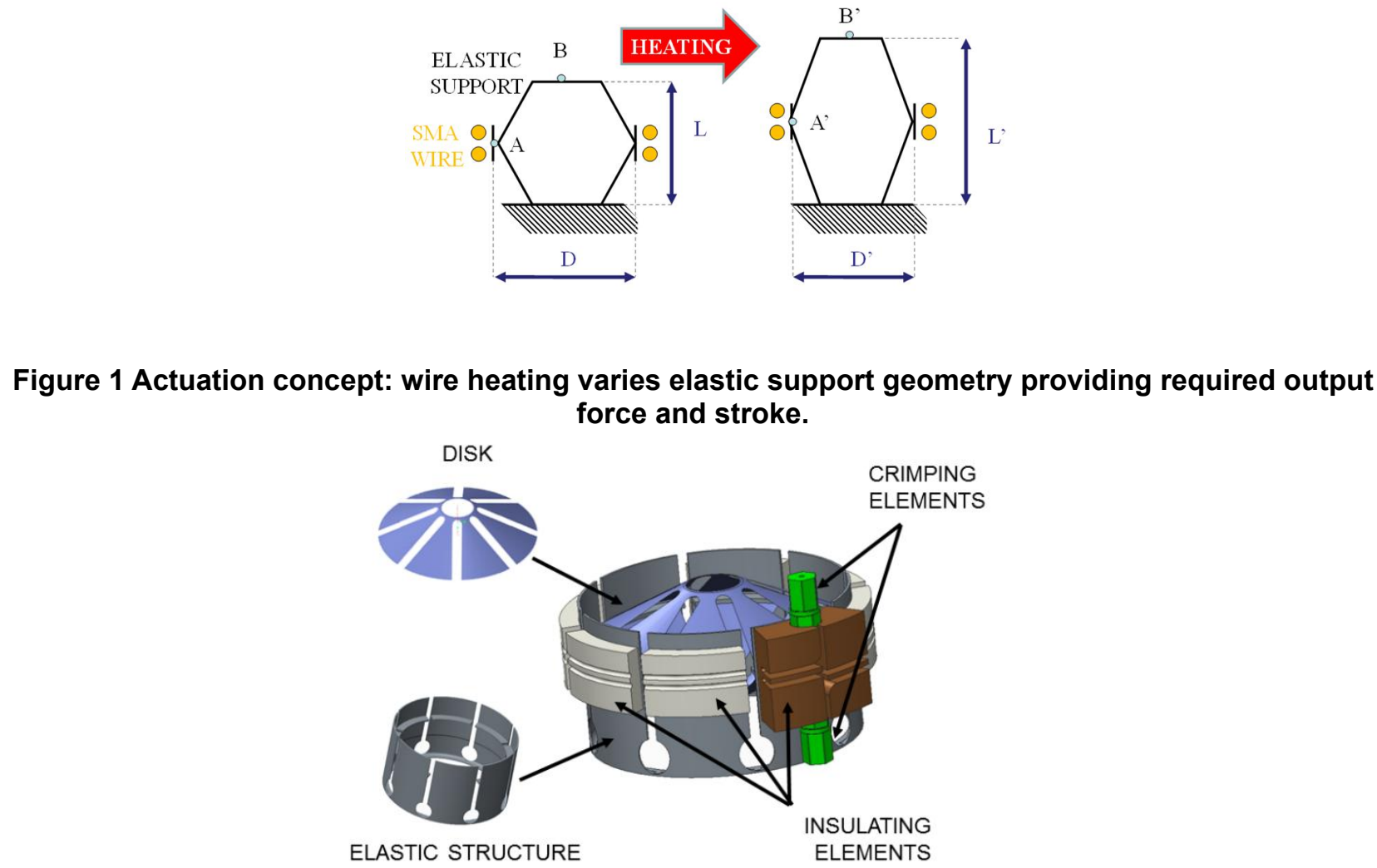

Figure 2 Single-shot actuator 3D model.

The system described in Figure 2 could be used alone as single shot actuator since it provides a stable open position once the SMA has been heated. However, no autonomous multiple actuations would be possible with this configuration because restoring of the initial position requires an external work. In resettable systems, this is achieved manually or in multiple actuations devices thanks to a bias spring that sets back the system when the SMA element cool down [15]. The latter configuration is simple and reliable but as previously mentioned, the need of power to keep the actuator in open configuration is often not acceptable, surely it would not be in our case where actuation would require 
117 almost the whole instrument allocated power. Thus, in our design two single shot actuators are

118 mounted in opposition. By facing two components of the type of that in Figure 1, and playing with

119 the alternate powering of the two actuators, the final configuration allows either amplification of the

120 output stroke and realization of the bidirectional actuator.

\subsection{Bidirectional actuator}

122 A section view of the designed bidirectional actuator is shown in Figure 3. Different elements are 123 present:

- a hold-down pin locking the spectrometer pendulum; and

- a bias spring, providing the required preload on the pendulum; it has to be noticed that the spring force is used only to provide the locking action and once the system is in open position, it gives a static load to be overcome by the actuator 1; and

- SMA wire wrapped around the insulating supports; number of wires turns was determined

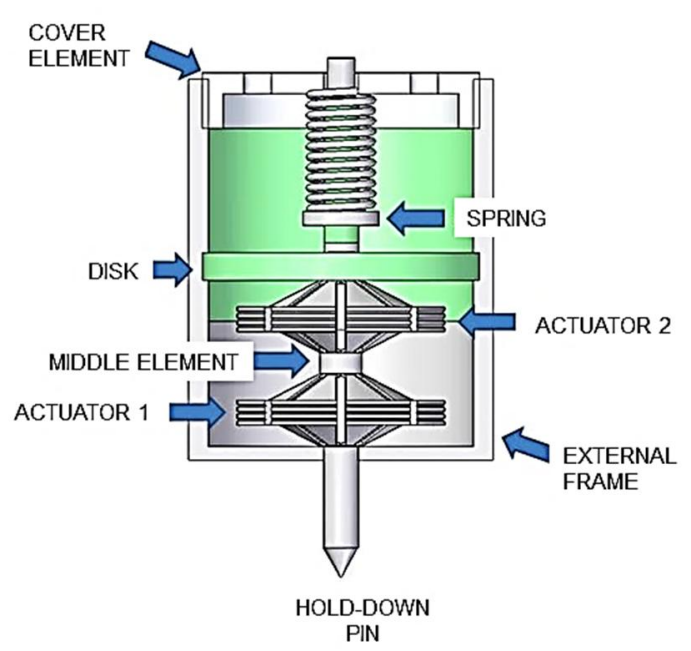


135 The two single-shot actuators (hereafter named actuator 1 and 2) are mounted in opposition to realize 136 the bidirectional configuration. Schematic of the intended opening/closing phases is shown in Figure 1374

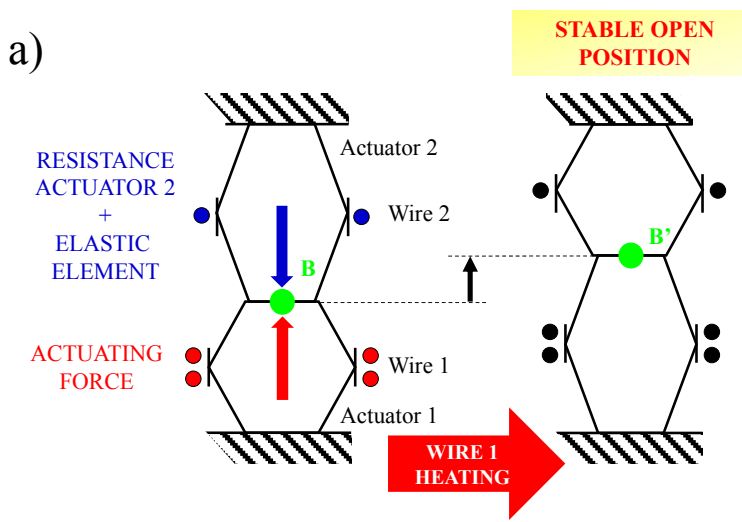

b)

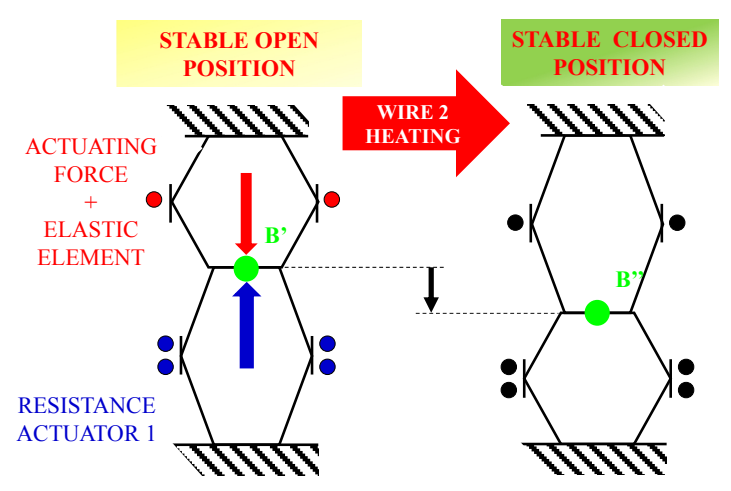

Figure 4 (a) Schematic of the actuation to achieve open stable position (b) backward actuation.

141 When actuator 1 is heated, the hold-down pin moves upwards. A complete release is possible only if 142 actuator 1 overcomes the spring bias force and stretches actuator 2 wire. Once heating of the actuator 1431 is switched off, the pin remains in the stable open position since the force provided by the bias 144 spring is lower than the one required to deform the wire 1. This is achieved by sizing the cross section 145 and number of wire turns of actuator 1.

146 Figure $4 \mathrm{~b}$ shows the locking procedure. The system starts in the stable open position as result of the 147 previous actuation. Wire 2 is heated, recovering its shape and providing the force required to move 148 back the system. Wire 2 heating is switched off when actuator 1 is completely stretched back and the 
hold-pin is locking the scanning mechanism. The bidirectional actuator is reset and ready for a new

150 cycle. In the following section detailed design of the single-shot actuator is provided. In particular, 151 disks geometry will be defined accounting for the motion phases and design requirements and the 152 single-shot actuator design will be optimized. To achieve required movement, actuator 1 and 2 153 comprise 2 and 1 turns of SMA wire, respectively.

\section{Thermo-mechanical design}

\subsection{Design requirements}

The actuator was originally developed for the Mars Infrared Mapper (MIMA)[22], a miniaturized infrared spectrometer payload of the 2007 configuration of the ExoMars high-mobility rover devoted to Mars surface observation. Unfortunately, a mission redesign aimed to mass and cost reductions stopped MIMA development since the instrument mineralogical and atmospheric science was regarded of minor importance with respect to the main exo-biologic mission's objectives. Despite that, MIMA was a lucky pick-up for the mechanism requirements definition; the extreme temperature range of the Martian environment, strict mass and power limitations of a rover mounted instrument, the strong dynamic loadings due to the landing phase, the cleanliness and shock-less request associated to the application on an interferometer, made it already compatible with any following proposed usage [23]. Design requirements for the single-shot actuator are summarized in the following:

- A linear displacement of $0.6 \mathrm{~mm}$ to unlock the instrument scanning pendulum; and

- a holding force of $50 \mathrm{~N}$, (required to warrant the locked position under the quasi-static acceleration of $1000 \mathrm{~m} / \mathrm{s}^{2}$ at landing on Mars); and

- mass (derived from the single-shot off-the-shelf actuator) of $15 \mathrm{~g}$ and volume limited to $40 \times 40 \times 20 \mathrm{~mm}^{3}$; and

- $7 \mathrm{~W}$ maximum power consumption; and 
- survival temperature range between -120 and $40{ }^{\circ} \mathrm{C}$, operating range between -80 and $40^{\circ} \mathrm{C}$;

\subsection{Kinematic model}

177 Forward kinematics allowed selection of the disk parameters to achieve required stroke, i.e. disk 178 external diameter and initial inclination angle. Disks shown in Figure 2 are symmetrical, therefore 179 two hinged rigid beams allow modelling the kinematic of each sector. The model is shown in 180 Figure 4. Moreover, considering that a symmetry exists also in the horizontal plane of the actuator, 181 the model is further simplified focusing on half actuator. Sketch of the simplified model is 182 provided in Figure 5.
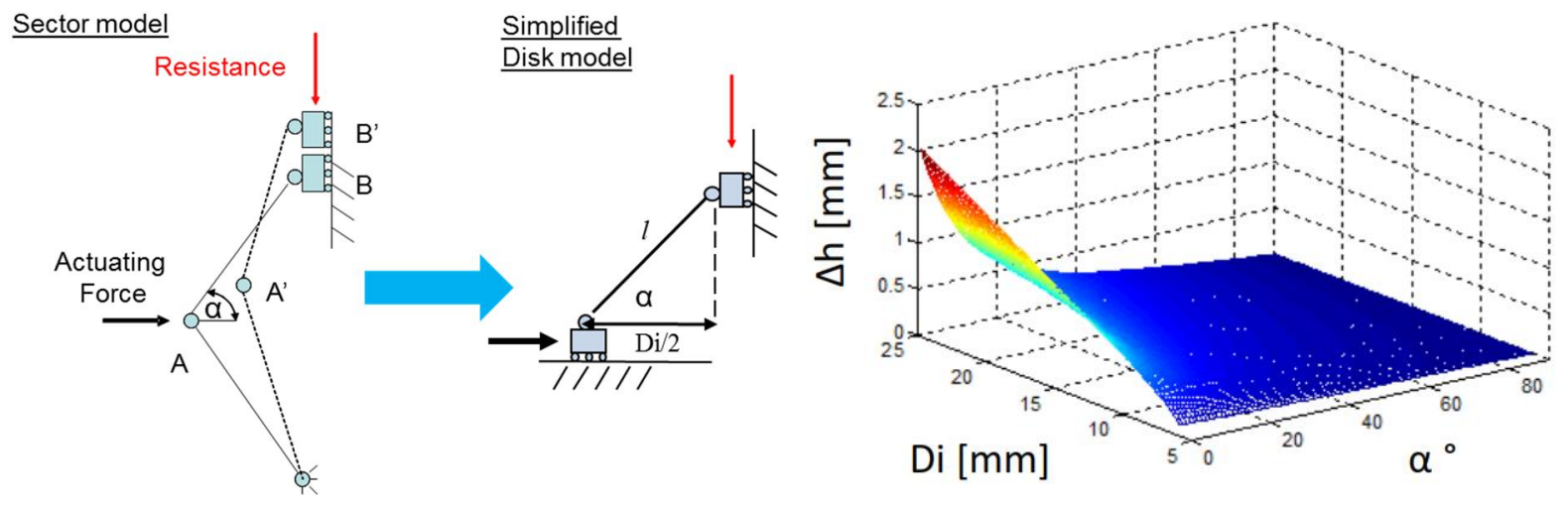

Figure 5 (Left) single shot actuator kinematic model (right) computed vertical displacement vs disk 187 size and initial inclination angle.

188 Knowing actuator geometry, i.e. inclination angle $\alpha$ and actuator diameter Di, vertical 189 displacement is obtained as: 
where $\Delta s$ is the actuator radial displacement that depends on the SMA wire contraction. As worst case, $\Delta s$ was restrained to $2 \%$ of the initial radius. Moreover, this value allows up to $10^{5}$ cycles of actuation considering the SMA aging with cycling. Besides, this limit well above the required value for the intended application and most locking devices. The static analyses performed are 195 bound to verify the feasibility of the proposed concept. In Figure 5, results of the kinematic analyses shows that by increasing the wrapping diameter the output stroke increases as well. Moreover, in order to maximize the amplification, disk inclination should be minimized. However, minimizing the angle reduces the force exerted on the pin especially at the beginning of the actuation. In fact, the force $\mathrm{V}$ (along the direction of the pin) and the radial one $\mathrm{H}$ (related to the 200 wire contraction), are linked by:

$201 \quad H=\frac{V}{\tan (\alpha)}$

202 where $\alpha$ is the inclination angle of the disk. Equation 2 has been used in the following to compute tensile stress on SMA material in the different actuation phases. The trade-off between output 204 stroke and force led in our case to define the preliminary disk geometry with an inclination angle 205 of $27^{\circ}$ and initial diameter of $25 \mathrm{~mm}$. Un-deformed disk diameter was set $28 \mathrm{~mm}$ and the disk was 206 divided in 8 sectors. The kinematic analysis evidenced that even in the worst case of minimum 207 wire contraction (i.e. $2 \%$ ), the actuator still provides the required output displacement of $0.6 \mathrm{~mm}$. 208 This result had been verified in the following by FE analyses on the actuator. Models have been 209 developed with PTC Creo Simulate software (C) PTC Inc.

\subsection{Disk design}

211 Disks design has been performed developing a FE model based on shell elements (elements 4782 212 and 4610 nodes) as shown in Figure 6. 


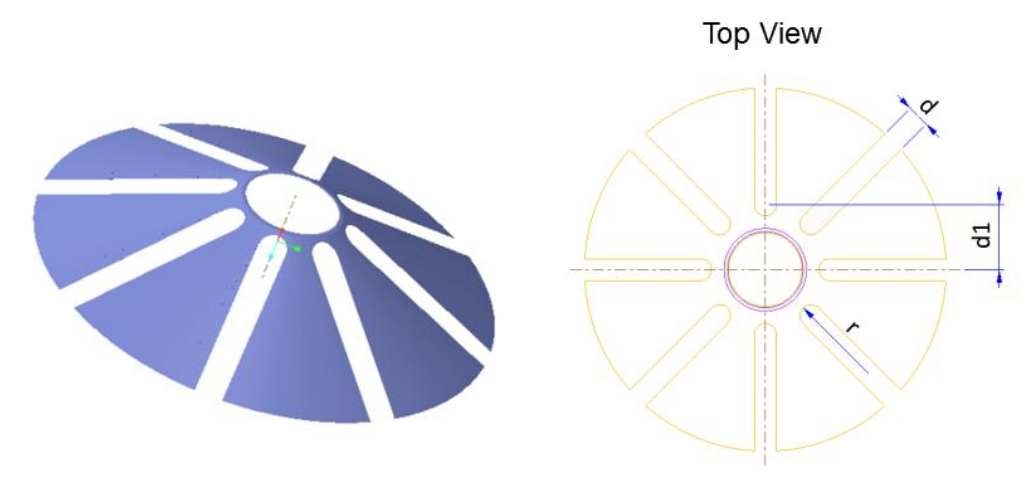

Figure 6 (Left) Disk FE model and (right) geometrical parameters.

216 Disk initial configuration has been selected by the simplified kinematic analyses presented above, 217 in which the disk sectors where considered as stiff elements. Disks thickness and cuts have to be 218 defined in order to completely define the disk geometry. Figure 6 shows remaining parameters to 219 be defined. In order to warrant resistance against expected loads in operative condition, buckling 220 analyses were performed. Maximum wire pull has been considered (i.e. $98 \mathrm{~N}$ ), considering 221 austenite plateau stress of manufacturer datasheet [24]. Half disk has been considered; 222 displacements in the horizontal plane and rotations have been left free for the external diameters 223 whereas hinge constraint has been applied at the inner disk circumference. Two different materials 224 have been considered, aluminum (A17075T6) and titanium (Ti6A14V) alloys. Material 225 characteristics are summarized in Table 1. Geometry of the disk has been varied in order to 226 minimize disk stiffness. This has been achieved with optimization analyses having set maximum 227 Von Mises stress for each material and minimum buckling coefficient, as for ECSS design [25].

\begin{tabular}{ccccc}
$\begin{array}{c}\text { Material } \\
\text { Property }\end{array}$ & Unit & Al 7075 T6 & Ti6Al4V & Macor \\
\hline Young Modulus & $\mathrm{GPa}$ & 70 & 114 & 66.9 \\
Density & $\mathrm{kg} / \mathrm{m}^{3}$ & 2800 & 4430 & 2520 \\
Poisson Ratio & & 0.33 & 0.33 & 0.29 \\
Ultimate Tensile Strength & $\mathrm{MPa}$ & 572 & 950 & 345 \\
Yield Tensile Strength & $\mathrm{MPa}$ & 380 & 880 & n.a. \\
thermal conductivity & $\mathrm{W} /(\mathrm{m} \mathrm{K})$ & 156 & 6.7 & 1.46 \\
\hline
\end{tabular}




\begin{tabular}{ccccc} 
& $\begin{array}{c}\text { Thickness } \\
{[\mathbf{m m}]}\end{array}$ & $\begin{array}{c}\text { d } \\
{[\mathbf{m m}]}\end{array}$ & $\begin{array}{c}\text { r } \\
{[\mathbf{m m}]}\end{array}$ & $\begin{array}{c}\text { d1 } \\
{[\mathbf{m m}]}\end{array}$ \\
\hline Optimization limits & 0.35 & 1 & 0.5 & 3.5 \\
& 0.6 & 2 & 1 & 5 \\
\hline Ti6A14V optimum & 0.35 & 1.5 & 0.75 & 4 \\
A17075T6 optimum & 0.38 & 1 & 0.5 & 4 \\
\hline
\end{tabular}
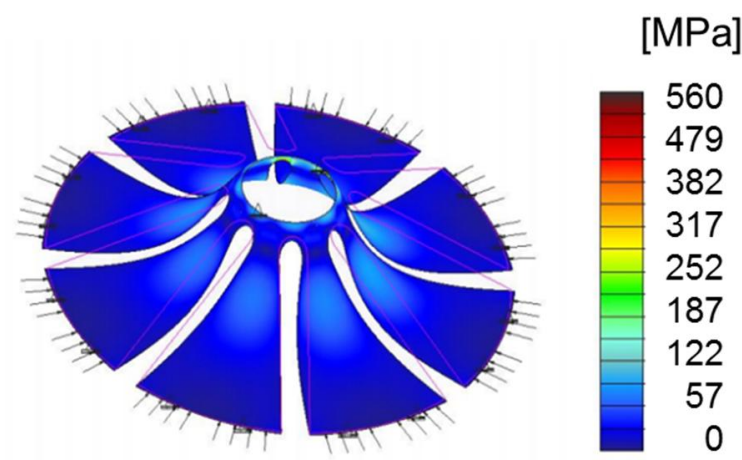

Figure 7 Von Mises stress over the titanium disk, $0.35 \mathrm{~mm}$ thickness.

235 It can be seen that the titanium alloy allows for thinner elements. This is expected because of the 236 higher mechanical strength and stiffness. In order to evaluate the force budget for the actuator 237 feasibility verification, stiffness of the disk has been evaluated by means of FE model. In fact, the 238 elastic deformation of the actuator due to the movement during wire contraction gives a resistance 239 to be overcome. Two contributions are evaluated; these are related to the deformation of the disks 240 once wire is recovering (upwards movement) or when a force from the top is applied (downwards 241 movement). The latter force is present when actuator 2 is positioning back the pin. Thus, a FE 242 model with two disks has been developed: disks have been matched with weighted links to allow 243 relative rotation of the disks during the simulated conditions. A static analysis has been performed, 244 with $1 \mathrm{~N}$ loading in radial direction. Deformed configurations is shown in Figure 8. In table 3 are summarized computed radial and vertical stiffnesses. 


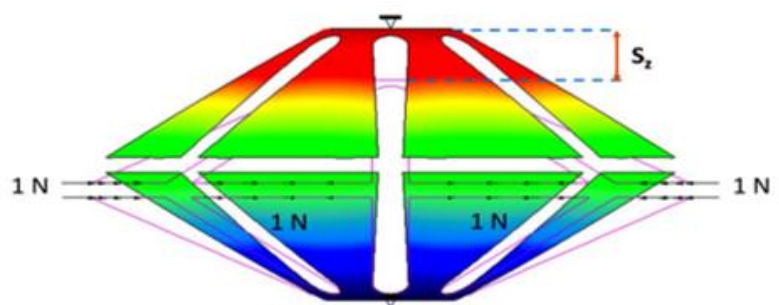

Figure 8 Disks deformation with radial loading of $1 \mathrm{~N}$.

\begin{tabular}{ccc} 
Material & $\begin{array}{c}\text { Radial } \\
\text { stiffness } \\
{[\mathbf{N} / \mathbf{m m}]}\end{array}$ & $\begin{array}{c}\text { Axial } \\
\text { stiffness } \\
{[\mathbf{N} / \mathbf{m m}]}\end{array}$ \\
\hline Ti6A14V & 5.15 & 10.26 \\
A17075T6 & 6.90 & 13.89
\end{tabular}

250 Comparison between candidate materials allowed evidencing that titanium alloy is the chosen 251 material thanks to the lower axial stiffness while providing larger safety margin during actuation.

252 It has to be reminded that a complete actuation is defined by three different phases:

- Opening phase: the pin puller unlock the pendulum and open position is achieved; beside the bias spring force, deformation of the disks and wire 2 deformation have to be overcome; and

- Static phase: the system is stuck and the movement is prevented by the resistance to deformation of wire 1; and

- Closing phase: the actuator 2 is activated, wire 1 is deformed again with the contribution of the force due to the bias spring; the actuator is reset and ready for the next actuation cycle.

According to ECSS design standard [25], friction force has to be considered with safety factor 3, elastic ones have to be multiplied or divided by 1.2 depending on the role of resistance or motor in the actuation. Forces can be computed for each phase and depend on the disk design, whose geometry 
264 and material have been selected on the basis of the performed analyses. Resulting stresses in the final

265 configuration for the wires of the actuator 1 and 2 are summarized in Table 4 . The detailed 266 computation of the stresses and the forces in each movement phase can be found in [26].

\begin{tabular}{ccc} 
Movement Phase & $\begin{array}{c}\text { Actuator 1 } \\
\text { [MPa] }\end{array}$ & $\begin{array}{c}\text { Actuator 2 } \\
\text { [MPa] }\end{array}$ \\
\hline Opening & 277.29 & 82 \\
Static & 59.92 & 0 \\
Closing & 82 & 335.98
\end{tabular}

268 The analysis evidence that actuation is feasible for each phase, so the external reset is no longer 269 needed. In fact, austenitic and martensitic phases plateau stresses specified by SMA wire 270 manufacturer, ranging between 70 and $650 \mathrm{MPa}$, are compatible with required tensile stresses during 271 each actuation phase. Anyway, the SMA wire selected for the actuator development underwent an 272 individual characterization, which is described in the following.

\subsection{Actuator design}

274 A FE model of the actuator 1 was built in order to verify the output displacement based on solid 275 tetrahedrons and shell elements (44047 elements and 36708 nodes). The FE model comprises the two 276 identical disks linked together, the elastic structure and the insulating supports. As previously 277 mentioned, the rotation between the disks border is allowed. Forces and constraints are shown in 278 Figure 9. Resistance load of $133.5 \mathrm{~N}$ is applied on the actuator and a radial force of $70 \mathrm{~N}$ resulting 279 from the wire pull has been distributed over the insulating sectors. 


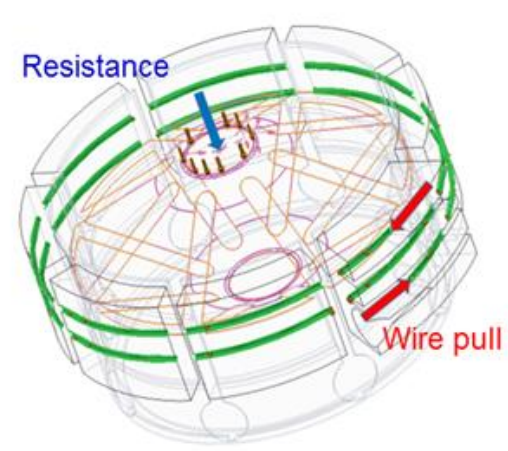

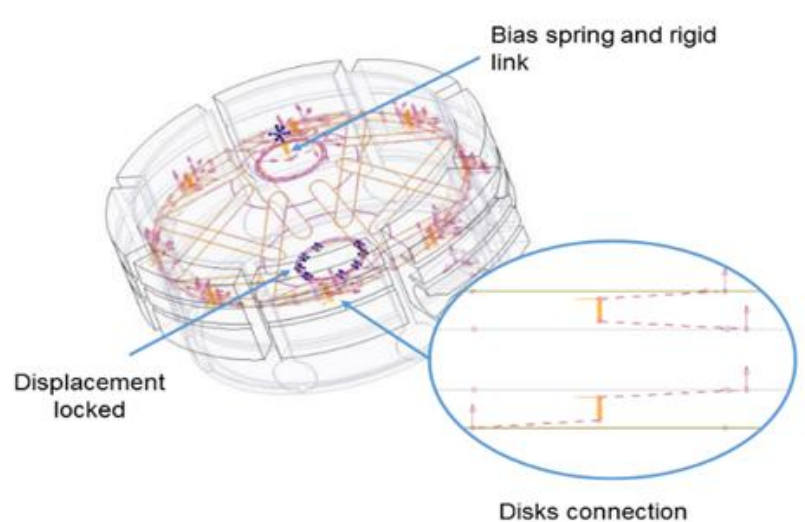

Figure 9 Actuator FE model (left), lumped elements and constraints (right).

The bias spring has been added on the top of the actuator, as well. A single-shot actuator has been optimized using as cost function the maximization of the output displacement [27, 28]; disk parameters in Figure 5 are left free. Figure 10 provides output displacement with optimal configuration and Table 5 summarizes optimized disk geometry.
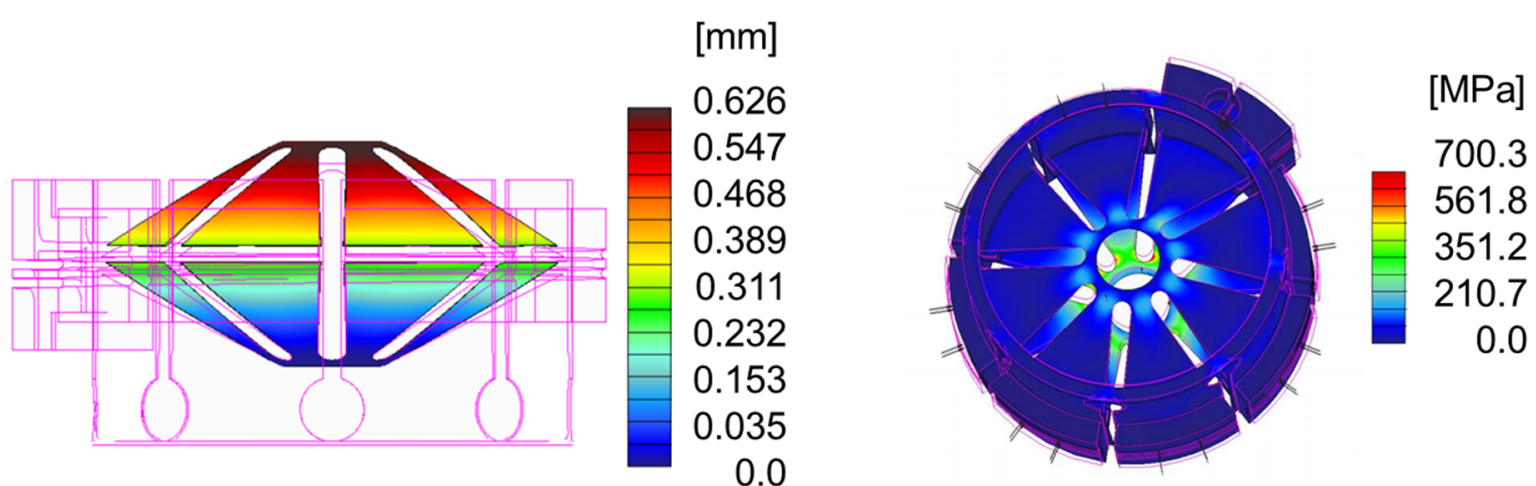

Figure 10 Actuator displacement (left) and Von Mises stress (right) during actuation phase.

\begin{tabular}{cccc}
$\begin{array}{c}\text { Thickness } \\
{[\mathbf{m m}]}\end{array}$ & $\begin{array}{c}\text { d } \\
{[\mathbf{m m}]}\end{array}$ & $\begin{array}{c}\mathbf{r} \\
{[\mathbf{m m}]}\end{array}$ & $\begin{array}{c}\text { d1 } \\
{[\mathbf{m m}]}\end{array}$ \\
\hline 0.283 & 1 & 0.5 & 4.08
\end{tabular}

\section{Table 5 Optimized single shot actuator parameters}

A thermal model of the actuator was developed with the aim of determining the power required to actuate the SMA wire in cryogenic condition. In fact, mission thermal analysis evidenced that in the case of cold Martian condition, expected lowest temperature is about $-120^{\circ} \mathrm{C}$ [23]. Actuators have 
been thermally linked together by means of null thermal resistance in order to simulate the worst case.

295 In fact, increasing the thermal resistance would reduce the power supply required for the actuation.

296 Moreover, radiation heat exchange has been imposed on the disks, insulating supports and wire. The 297 disks emissivity has been set to 0.05 , assuming a gold coating of the titanium disks while unitary 298 emissivity has been assumed for the insulating ceramic supports. In order to reduce the model 299 complexity, wires have been modelled by separated circles on which heating has been uniformly 300 distributed, simulating Joule heating effect. The two extreme conditions "cold" and "hot" have been 301 analyzed; radiative and conductive interfaces have been set to $-120^{\circ} \mathrm{C}$ and $40^{\circ} \mathrm{C}$, respectively. The 302 predicted temperature distributions in cold and hot cases are shown in Figure 11. The transformation 303 temperature of $90^{\circ} \mathrm{C}$ is achieved on actuatorl heating the wire with 1.86 and $0.62 \mathrm{~W}$, for cold and hot 304 case respectively. These values are fully compliant with the mission requirements. Moreover, it can 305 be seen that in the simulated cases temperature of the wire 2 is always by far than the one required for 306 complete austenite transformation, so there is no risk of simultaneous actuation of the two wires that 307 would lead to a failure.

308 Final configuration for the single shot actuator has $7 \mathrm{~g}$ mass and radial size of $34 \mathrm{~mm}$ and height of $30915 \mathrm{~mm}$.

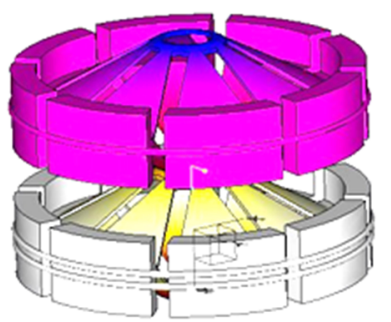

310 $\left[{ }^{\circ} \mathrm{C}\right]$

91.3 


\section{Experimental activity}

\subsection{Wire characterization}

315 SMA wire from Memory-metalle $\mathrm{GmbH} / \mathrm{Saes}$ Group has been purchased for the actuator 316 development. Material characteristics have been measured to identify the transformation 317 temperatures in operative conditions and the stress-strain curves for the material different phases.

318 DSC Seiko calorimeter model 220 has been used for the calorimetric characterization. DSC samples

319 with $20 \mathrm{mg}$ mass have been tested between -50 and $150^{\circ} \mathrm{C}$. Temperature variation has been performed 320 with $10{ }^{\circ} \mathrm{C} / \mathrm{min}$ rate and three cycles have been done. Specific heat flux curves are provided in Figure 32112.

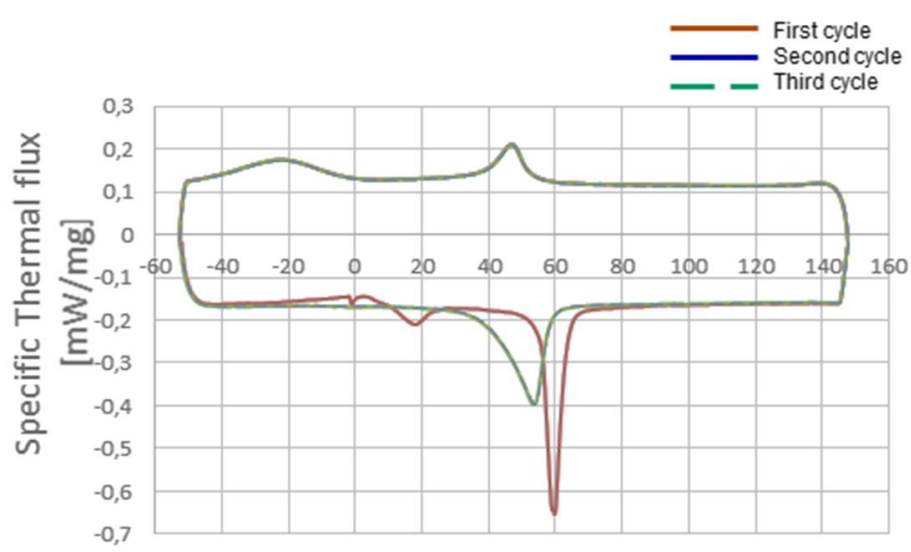

Temperature $\left[{ }^{\circ} \mathrm{C}\right]$

Figure 12 Results of the DSC testing.

MTS testing facility allowed functional testing to determine stress-strain curves in the martensitic and austenitic phases. Material austenitic and martensitic states had been obtained by means of thermal 327 chamber at high (about $100{ }^{\circ} \mathrm{C}$ ) and low (about RT) temperatures respectively. Tensile loading/unloading cycles were performed, at constant temperatures, with $25 \mathrm{~mm}$ strain gauge extensometer and a preload of $2 \mathrm{~N}$. Deformation rate was set to $2 \mathrm{~mm} / \mathrm{min}$ with maximum deformation 330 of $3 \%$. Temperature variation between 22 and $24{ }^{\circ} \mathrm{C}$ was accepted for the martensite testing whereas $33195^{\circ} \mathrm{C}$ was the kept as minimum for the austenitic phase characterization. Results of the tensile tests 332 are shown in Figure 13. 


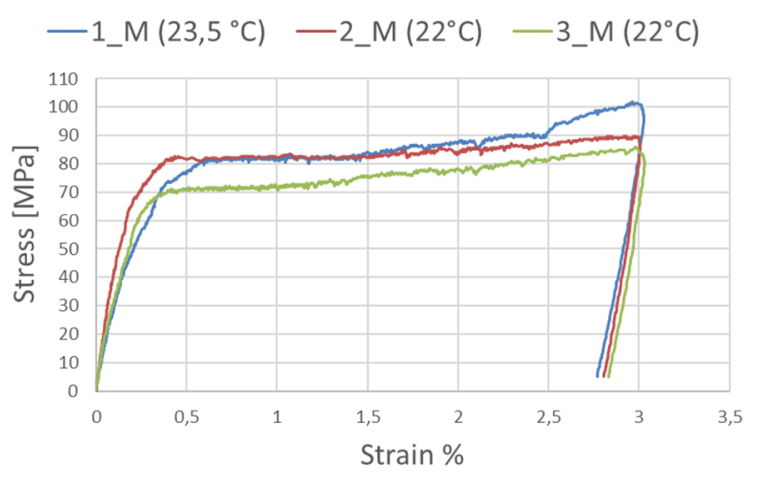

333

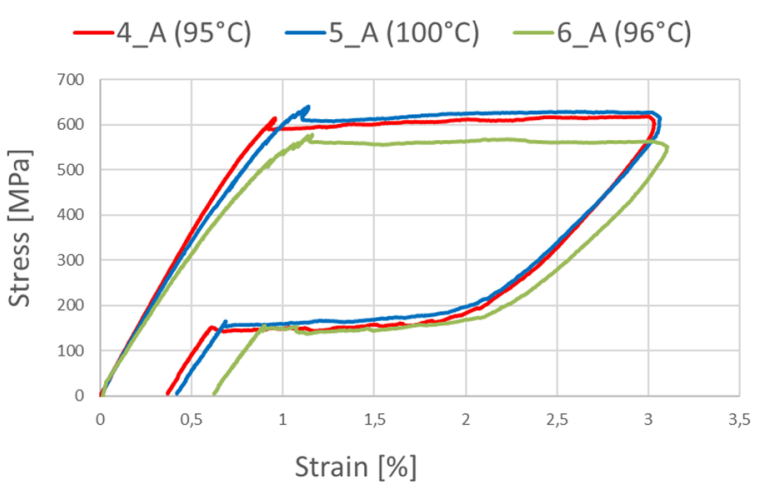

Figure 13 Functional testing: martensitic (left) and austenitic (right) states.

Table 6 provide mechanical characteristics derived from stress-strain curves.

\begin{tabular}{ccc} 
Test ID & Elastic Modulus [GPa] & Plateau Stress [MPa] \\
\hline 1_M & 28 & 80 \\
2_M & 43 & 80 \\
3_M & 30 & 70 \\
4_A & 73 & 602 \\
5_A & 70 & 628 \\
6_A & 63 & 560
\end{tabular}

Table 6 DSC and functional testing transformation temperatures. Units are ${ }^{\circ} \mathrm{C}$.

Strain recovery heating/cooling loops, under constant load (simulating working condition with dead mass of $5 \mathrm{~kg}$, i.e. nominal tensile stress of $250 \mathrm{MPa}$ ) and temperature cycling has been performed in a thermal chamber (ACS Angenlantoni Industrie type), equipped with a LVDT (Linear variable differential transducer) to measure the wire deformation under heating and cooling. Temperature variation rate was set to $2^{\circ} \mathrm{C} / \mathrm{min}$ between -50 and $150{ }^{\circ} \mathrm{C}$ and 10 cycles between minimum and maximum temperatures had been performed. Results are shown in Figure 14. 


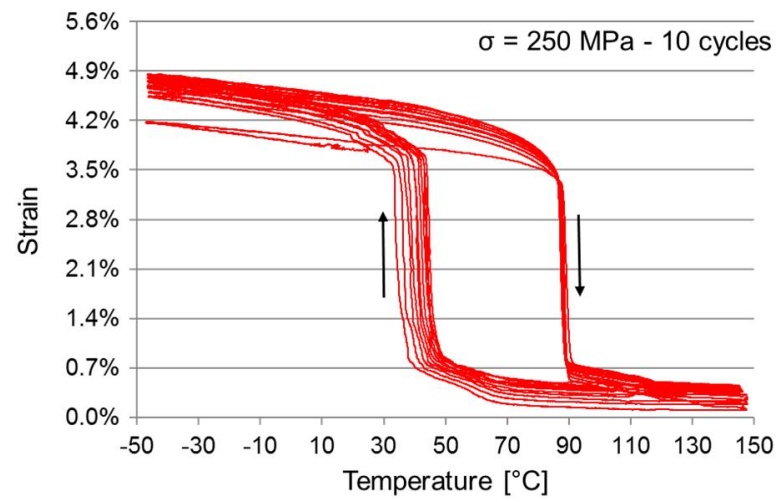

Figure 14 Functional testing: strain vs temperature.

347 Obtained transformation temperatures are compared with DSC results in Table 7.

\begin{tabular}{ccccc} 
Temperatures & DSC & $\mathbf{1}^{\text {st }} \mathbf{c y c l e}$ & $\mathbf{2}^{\text {nd }} \mathbf{c y c l e}$ & $\mathbf{1 0}^{\text {th }}$ cycle \\
\hline Ms & -3.6 & 38.46 & 38.51 & 46.52 \\
Mf & -45 & 32.34 & 35.68 & 43.20 \\
As & 39 & 87.31 & 87.07 & 85.74 \\
Af & 57 & 89.63 & 88.61 & 87.66
\end{tabular}

\subsection{Discussion}

350 Heat peaks in Figure 12 identify phase transformation temperatures. During cooling two peaks have 351 been found, since between martensite and austenite the phase $\mathrm{R}$ is present. During heating just one 352 peak is found since the transformation peaks are coincident. Transformation temperatures, 353 summarized in Table 7, have been derived looking to the intersection between the tangent of the DSC 354 curves at the measured peaks. Austenitic phase is achieved at relatively low temperature, about 60 $355{ }^{\circ} \mathrm{C}$. This is important for our design, since a low heating power is expected even in cryogenic 356 condition (as confirmed by thermal modelling). As expected, for a non-trained wire, there is not a 357 thermal cycling stability particularly for the firsts heating/cooling cycles (see DSC cooling curves) 358 [29]. However, after three DSC scans a stabilization is achieved. Typical thermal hysteresis (about $35940^{\circ} \mathrm{C}$ ) of NiTi wire for shape memory application is shown also. 
360 Figure 13 depicts mechanical behaviors of the material and the characteristic plateaus related to the

361 de-twinning of the martensite (low temperature) and stress induced martensite (high temperature) are 362 well shown. Mechanical modulus as well as stresses plateau values are summarized in Table 6. It can 363 be seen that measured values are compatible with datasheet specifications and are in agreement with 364 previous literature studies. For the martensitic phase the elastic modulus varies between 28 and 43 $365 \mathrm{GPa}$ and plateau stress is varying between 70 and $80 \mathrm{MPa}$. For the austenitic phase, the elastic 366 modulus and plateau stress increase up to $70 \mathrm{GPa}$ and $600 \mathrm{MPa}$, respectively. Worst case combination 367 of the measured mechanical properties had been used in the feasibility and optimization design 368 phases.

369 Finally, except for the first cycle, the wire under constant load of $250 \mathrm{MPa}$ exhibits a stable functional 370 performance after few heating/cooling cycles (see Figure 14). This means that with proper training 371 (lasting no more than 10 cycles), the wire is ready for the actuation. Another important behavior is 372 that the transformation temperatures change as consequence of the applied stress. Table 7 evidences 373 that a general increase of the temperature is obtained. In particular, $\mathrm{M}_{\mathrm{f}}$ is about $40^{\circ} \mathrm{C}$ and $\mathrm{A}_{\mathrm{f}}$ achieves $37487^{\circ} \mathrm{C}$. The latter values had been used as reference for the design described in Section 2.

\subsection{Actuator Testing}

376 With the aim of verifying the design and highlighting drawbacks and possible improvements, a 377 mock-up for the single actuator has been realized. Figure 15 shows the single-shot actuator 378 breakdown and the experimental setup. 

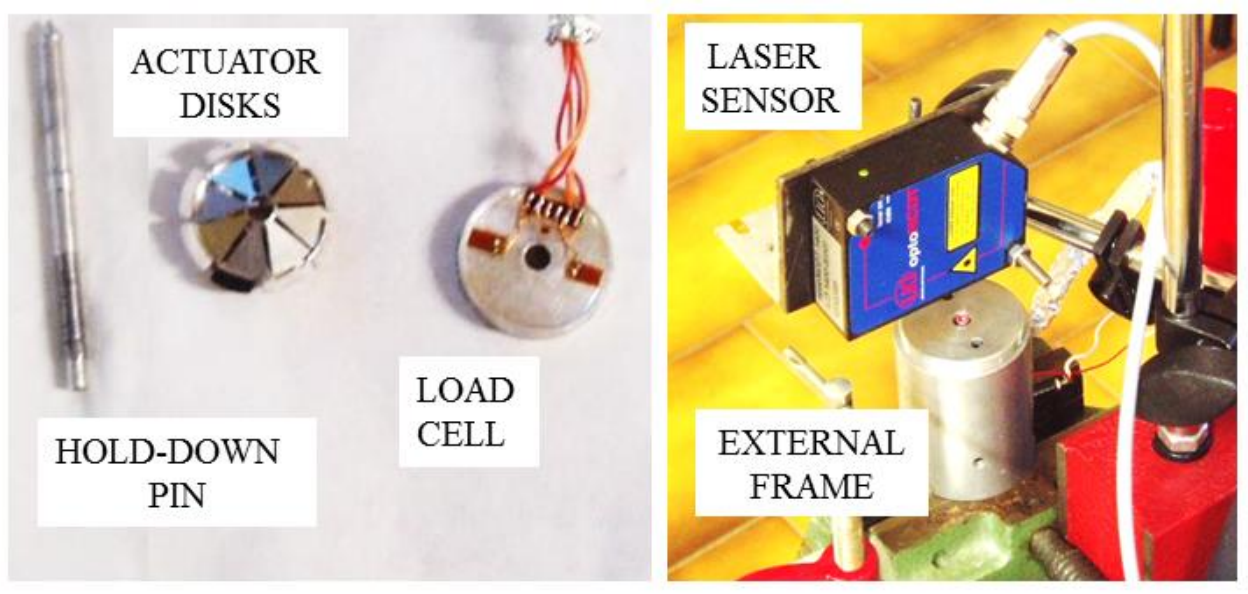

Figure 15 Actuator breakdown, load cell and experimental setup for the actuator testing.

Actuating force was measured by a load cell (range $150 \mathrm{~N}$, linearity $0.5 \%$ of the measurement range) mounted between the hold-down pin and the ground. Displacement has been measured by laser Micro Optronic ILD 1400-05 (range 5mm, maximum linearity error $9 \mu \mathrm{m}$ ). The single-shot actuator was loaded with a dead mass of $10 \mathrm{~kg}$ (as worst case loading) and powered with a constant current of $1.8 \mathrm{~A}$, value was selected to achieve complete transformation but avoid the wire overheating. Figure 16 shows measured force and displacement during an actuation cycle.

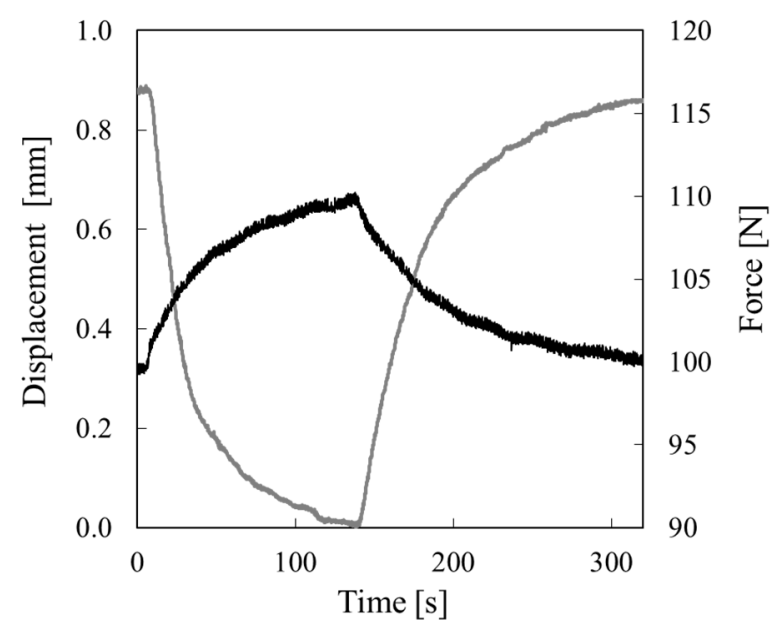

Figure 16 Measured displacement (grey color) and force (black) of the single actuator.

Once that the actuation starts, the hold-down pin reaches the maximum displacement of $0.9 \mathrm{~mm}$ after about $140 \mathrm{~s}$. This is confirmed by the load increasing, due to the spring element, up to 110 N. 
Once the electrical power is switched off, the system comes back to the initial position in about

395 three minutes. This is expected since the load is larger than the one that causes system closing. 396 Moreover, the measured stroke is higher than the nominal $0.6 \mathrm{~mm}$. This result was expected too, 397 because the actual SMA recovery was larger than the $2 \%$ value, assumed as end of life figure in 398 the design. This happened despite the wire had been "aged" with more than 1000 cycles. The obtained result evidenced the need of an accurate control of the actuator geometry and wire contraction, to match the expected performances. As positive outcome, the obtained result also highlighted the intrinsic flexibility of the proposed concept that can be adjusted to different displacement requirements with small changes of the starting wire length.

\section{Conclusions}

A light and small bidirectional actuator was designed to lock the mechanism of a miniaturized infrared spectrometer. The actuator is based on SMA technology and can provide $0.6 \mathrm{~mm}$ output displacement against $50 \mathrm{~N}$ loading. The actuator is based on elastic structures whose geometry can be changed to face different requirements in terms of force or displacement, which amplify the contraction of the SMA wire. The bidirectional working is achieved without requiring power either than that necessary

410 for the position commutation. Moreover, the actuator concept flexibility can be exploited to match 411 different working condition, i.e. by changing the wire diameter to achieve higher forces or the actuator 412 geometry to allow larger displacements. A commercially available wire has been selected and tested 413 to develop a mockup of the proposed actuator. Testing of the manufactured mockup allowed 414 preliminary validation of the proposed concept. Future activity is foreseen to fully characterize the 415 designed actuator in terms of force vs displacement performance in more general working conditions; this would enable evaluating the actuator suitability for applications different from the designed 417 locking system. 
1. J. M. Jani, M. Leary, A. Subic, and M. A. Gibson, "A review of shape memory alloy research, applications and opportunities," Mater Des 56, 1078-1113 (2014).

2. J. Sheng and J. P. Desai, "Design, modeling and characterization of a novel meso-scale SMAactuated torsion actuator," Smart Mater. Struct. 24, 105005 (2015).

3. D. Patil and G. Song, "Shape memory alloy actuated accumulator for ultra-deepwater oil and gas exploration," Smart Mater. Struct. 25, 045012 (2016).

4. S. Barbarino, E. S. Flores, R. Ajaj, I. Dayyani, and M. Friswell, "A review on shape memory alloys with applications to morphing aircraft," Smart Mater. Struct. 23, 063001 (2014).

5. A. Razov and A. Cherniavsky, "Applications of shape memory alloys in space engineering: past and future," EUROPEAN SPACE AGENCY-PUBLICATIONS-ESA SP 438, 141-146 (1999).

6. E. M. Flint, J. Melcher, and H. Hanselka, "The "promise'of smart materials for small satellites," Acta Astronaut. 39, 809-814 (1996).

7. H. Oh, S. Jeon, T. Kim, and Y. Kim, "Experimental feasibility study for micro-jitter attenuation of stepper-actuated X-band antenna-pointing mechanism by using pseudoelastic SMA mesh washer," Smart Mater. Struct. 24, 045010 (2015).

8. A. Peffer, K. Denoyer, E. Fosness, and D. Sciulli, "Development and transition of low-shock spacecraft release devices," in Aerospace Conference Proceedings, 2000 IEEE, Anonymous (IEEE, 2000), pp. 277-284.

9. T. Georges, V. Brailovski, and P. Terriault, "Characterization and design of antagonistic shape memory alloy actuators," Smart Mater. Struct. 21, 035010 (2012).

10. F. Peng, X. Jiang, Y. Hu, and A. Ng, "Actuation precision control of SMA actuators used for shape control of inflatable SAR antenna," Acta Astronaut. 63, 578-585 (2008).

11. M. Lee, J. Son, H. Hwang, Y. Kim, and B. Kim, "Shape Memory Alloy (SMA) Actuator Based Separation Device," (2011).

12. Y. I. Yoo, J. W. Jeong, J. H. Lim, K. Kim, D. Hwang, and J. J. Lee, "Development of a nonexplosive release actuator using shape memory alloy wire," Rev. Sci. Instrum. 84, 015005 (2013).

13. R. Edeson, M. Whalley, B. Kent, S. Canfer, and E. Sawyer, "SMA gas release mechanism for the Rosetta Lander's Ptolemy instrument," Acta Astronaut. 58, 576-582 (2006).

14. O. Benafan, R. Noebe, and T. Halsmer, "Static rock splitters based on high temperature shape memory alloys for planetary explorations," Acta Astronaut. 118, 137-157 (2016).

451 15. Q. Donnelan, "Design and testing of linear shape memory alloy actuator," Final Report, 452 National Science Foundation-Research Experience for Undergraduates (2005). 
16. B. Saggin, E. Alberti, L. Comolli, M. Tarabini, G. Bellucci, and S. Fonti, "MIMA, a miniaturized infrared spectrometer for Mars ground exploration: Part III. Thermomechanical design," in Remote Sensing, Anonymous (International Society for Optics and Photonics, 2007), pp. 67441S-67441S-10.

17. I. Shatalina, B. Saggin, D. Scaccabarozzi, R. Panzeri, and G. Bellucci, "MicroMIMA FTS: design of spectrometer for Mars atmosphere investigation," in SPIE Remote Sensing, Anonymous (International Society for Optics and Photonics, 2013), pp. 88900T-88900T-12.

18. B. Saggin, D. Scaccabarozzi, I. Shatalina, R. Panzeri, M. Tarabini, M. Magni, and G. Bellucci, "MicroMIMA, a miniaturized spectrometer for planetary observation," in Metrology for Aerospace (MetroAeroSpace), 2015 IEEE, Anonymous (IEEE, 2015), pp. 502-506.

19. H. Funakubo, "Shape Memory Alloys,(1987)," Gordon and Breach Science Pub1-60 (2008).

20. H. Ishii and K. Ting, "SMA actuated compliant bistable mechanisms," Mechatronics 14, 421437 (2004).

21. G. S. Mammano and E. Dragoni, "Increasing stroke and output force of linear shape memory actuators by elastic compensation," Mechatronics 21, 570-580 (2011).

22. B. Saggin and D. Scaccabarozzi, "Design and optimization of the calibration procedure for a miniaturized Fourier transform spectrometer," Appl. Spectrosc. 65, 627-633 (2011).

23. D. Scaccabarozzi, B. Saggin, and E. Alberti, "Design and testing of a roto-translational shutter mechanism for planetary operation," Acta Astronaut. 93, 207-216 (2014).

24. Memory Metalle GmbH, "Info Sheet No 4, Selected Properties of NiTi-based Alloys, http://www.memory-metalle.de/html/03 knowhow/PDF/MM_04_properties_e.pdf," .

25. ESA, "ECSS E30 Part 2 A, Mechanical—Part 2: Structural," .

26. G. Balatti, "Sviluppo di un attuatore bidirezionale per applicazione spaziale, MSc degree, Polytechnic University of Milan, https://www.politesi.polimi.it/handle/10589/109009," (2015).

27. M. G. Gritti, H. Giberti, and A. Collina, "Optimal synthesis of a cam mechanism for train pantograph," in Mechatronics (ICM), 2013 IEEE International Conference on, Anonymous (IEEE, 2013), pp. 406-411.

28. H. Giberti, S. Cinquemani, and S. Ambrosetti, "5R 2dof parallel kinematic manipulator-A multidisciplinary test case in mechatronics," Mechatronics 23, 949-959 (2013).

29. A. Tuissi, P. Bassani, A. Mangioni, L. Toia, and F. Butera, "Fabrication process and characterization of NiTi wires for actuators." in SMST-2004: Proceedings of the International Conference on Shape Memory and Superelastic Technologies, Anonymous (, 2006), pp. 501-508. 